\title{
Performance of Prognostic Risk Scores in Elderly Chinese Patients with Heart Failure
}

\author{
Yalin Cheng \\ Ke Chai \\ Wanrong Zhu \\ Yuhao Wan \\ Yaodan Liang \\ Minghui Du \\ Yingying Li \\ Ning Sun \\ Jiefu Yang \\ Hua Wang (DD
}

Department of Cardiology, Beijing Hospital, National Center of Gerontology, Institute of Geriatric Medicine, Chinese Academy of Medical Sciences, Beijing, People's Republic of China
Correspondence: Hua Wang Department of Cardiology, Beijing Hospital, National Center of Gerontology, Institute of Geriatric Medicine, Chinese Academy of Medical Sciences, Beijing, People's Republic of China

Tel/Fax +86-10-85133852

Email wanghua2764@bjhmoh.cn
Purpose: Elderly heart failure (HF) patients have different clinical characteristics and poorer prognosis compared with younger patients. Prognostic risk scores for HF have not been validated well in elderly patients. We aimed to validate the Seattle Heart Failure Model (SHFM) and the Meta-Analysis Global Group in Chronic Heart Failure (MAGGIC) score in an elderly Chinese HF cohort.

Patients and Methods: This retrospective study enrolled 675 elderly HF patients (age $\geq 70$ years) discharged from our hospital between 2012 and 2017. The performance of the two risk scores was evaluated in terms of discrimination, using receiver-operating characteristic analysis, and calibration using a calibration plot and Hosmer-Lemeshow (H-L) test. Absolute risk reclassification was used to compare the two scores.

Results: During the mean follow-up time of 32.6 months, 193 patients (28.6\%) died, and 1 -year mortality was $10.5 \%$. The predicted median 1 -year mortality was $8 \%$ for the SHFM and $18 \%$ for the MAGGIC score. A Kaplan-Meier survival curve demonstrated that event rates of all-cause mortality significantly increased with increasing SHFM and MAGGIC scores. The discriminatory capacity of the SHFM was greater than that of the MAGGIC score (c-statistics were 0.72 and 0.67 , respectively; $\mathrm{P}=0.05$ ). The calibration plot for the SHFM was better than that for MAGGIC score for 1-year mortality (SHFM: H-L $\chi^{2}=8.2$, $\mathrm{P}=0.41$; MAGGIC: H-L $\left.\chi^{2}=18.8, \mathrm{P}=0.02\right)$. Compared with the MAGGIC score, the net reclassification index (NRI) of the SHFM was $2.96 \%(\mathrm{Z}=5.88, \mathrm{P}<0.0001)$.

Conclusion: The SHFM performs better than MAGGIC score, having good discrimination, calibration and risk classification for the prediction of 1-year mortality in elderly Chinese HF patients.

Keywords: heart failure, risk score, prognosis, elderly patient

\section{Introduction}

Heart failure (HF) is a growing health problem worldwide, especially in the elderly. ${ }^{1}$ The prevalence of HF increases from $0.9 \%$ in people aged $55-64$ years to $17.4 \%$ in those aged 85 years and over. ${ }^{2}$ The 1 -year mortality risk increases with advancing age by $60 \%$ per decade. ${ }^{3,4}$ Despite recent improvements in medications and device therapy, ${ }^{5}$ the benefits to the elderly remain uncertain because they are underrepresented in clinical trials. Compared with younger patients, elderly patients are more likely to experience HF with preserved ejection fraction (HFpEF), have a greater burden of co-morbidities, and unique problems with medication tolerance. ${ }^{6,7}$ Considering the distinct features and poor prognosis of elderly patients, targeted risk assessment and treatment strategies need to be developed specifically for the elderly. 
Accurate assessment of prognosis is critical to improving patient care and optimizing medical resource allocation. ${ }^{8}$ Several prognostic models have been established to evaluate long-term mortality of HF patients. ${ }^{9}$ The Seattle Heart Failure Model (SHFM) and Meta-Analysis Global Group in Chronic Heart Failure (MAGGIC) score are two of these risk metrics with good discrimination and calibration. Both models have been thoroughly validated and there are online calculators for clinical use. ${ }^{10,11}$ However, evidence supporting the use of these risk models for elderly patients is limited. In this study, we externally validated and compared the SHFM and MAGGIC scores with respect to their ability to predict 1-year mortality in an elderly Chinese HF cohort.

\section{Patients and Methods}

\section{Study Population}

This was a retrospective observational study. Patients aged 70 years or older with a diagnosis of HF who had been discharged from the cardiology department of Beijing Hospital between January 2012 and December 2017 were enrolled consecutively. These patients included symptomatic HF patients admitted for decompensated or chronic HF in the stable period, or admitted for related reasons such as unstable angina or hypertension. All patients were recruited as outpatients after discharge. HF diagnosis was made by experienced cardiologists using the clinical practice guidelines of the Chinese Society of Cardiology, and was based on (i) symptoms, for example, dyspnea, fatigue, or decreased exercise capacity; (ii) signs, for example, edema or rales; (iii) B-type natriuretic peptide or N-terminal pro-B-type natriuretic peptide, to differentiate HF diagnosis for patients with dyspnea; and (iv) structural and functional abnormalities observed by echocardiography. ${ }^{12}$ The patient flowchart is shown in Figure 1. We included HF patients with reduced EF (HFrEF, EF $<40 \%$ ), moderate EF (HFmrEF, EF: 40$49 \%$ ), and preserved $\mathrm{EF}$ (HFpEF, $\mathrm{EF} \geq 50 \%$ ). Data was extracted from the patients' electronic records to calculate the SHFM and MAGGIC scores. Patients who were lacking any components of the SHFM or the MAGGIC score ( $\mathrm{n}=$ 18), had cancer at baseline $(\mathrm{n}=112)$, or were unavailable for follow-up $(n=133)$, were excluded. Finally, we included 675 HF patients aged 70 years or older for analysis. All study participants signed the informed consent agreement. The study conformed to the Declaration of Helsinki and was approved by the ethics review board of Beijing Hospital (2018BJYYEC-059-02).

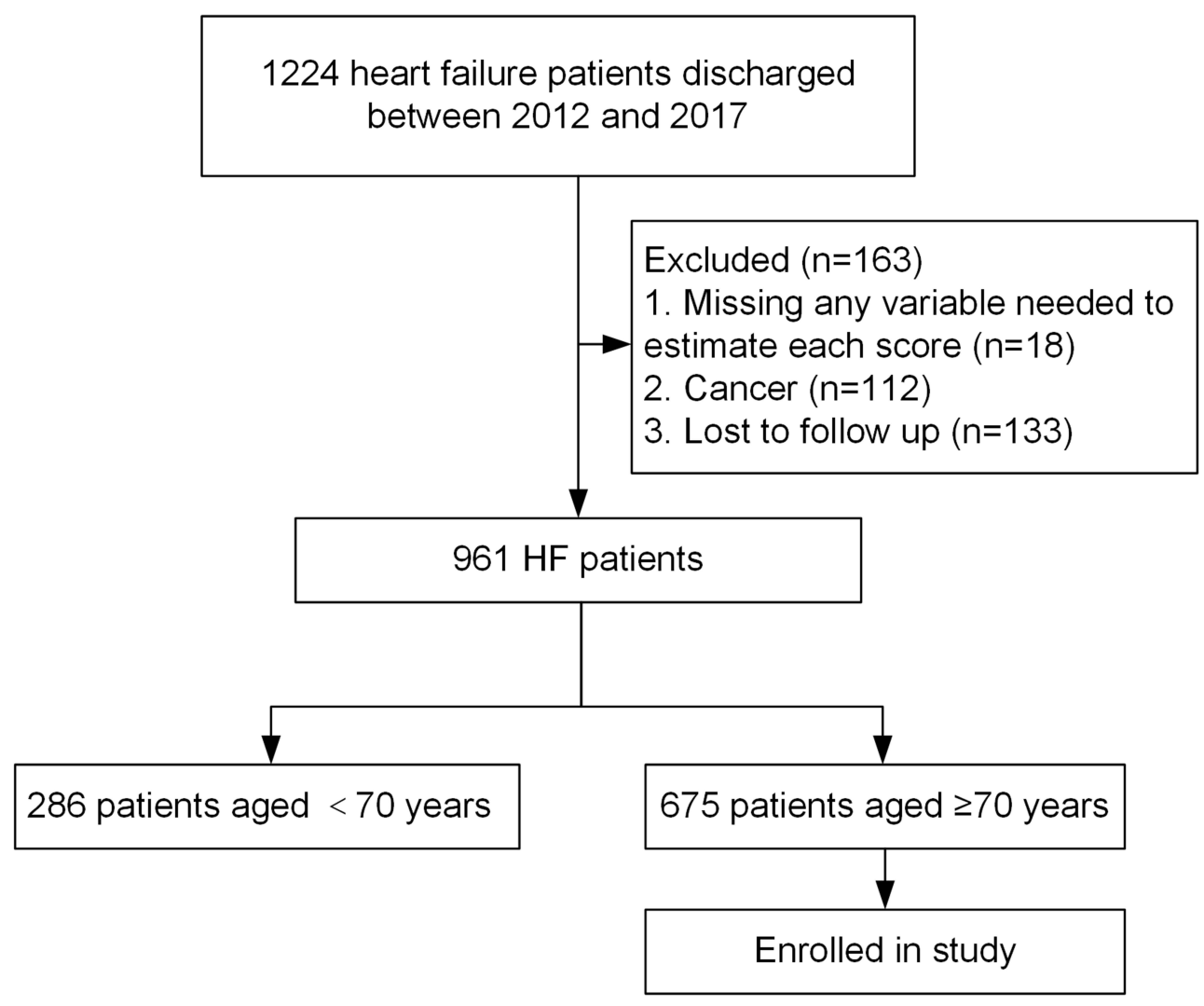

Figure I Patient flowchart of this study. 


\section{Risk Scores}

We used four criteria to select the risk scores: 1) risk scores designed to predict 1-year all-cause mortality; 2) data needed to calculate the risk scores were routinely available and did not contain results of cardiopulmonary exercise tests; 3 ) risk scores were externally validated with good discrimination (c-statistic $>0.65$ ); 4) risk scores were validated in HF patients discharged from the hospital. ${ }^{13,14}$ Finally, the SHFM and the MAGGIC score were selected.

A detailed list of the variables included in both models is provided in Table 1. Predicted 1-year mortality was calculated using the online SHFM (https://depts.washington.edu/shfm) and MAGGIC score (http://www.heartfailurerisk.org). ${ }^{10,11}$

\section{Follow-Up and Outcomes}

The primary outcome was 1-year mortality. One-year survival status was ascertained by examination of hospital and outpatient department records and death certificates. If there were no records, we would conduct a telephone interview with the patient or his/her family.

\section{Statistical Analysis}

The characteristics of all patients at baseline are described by means and SDs for continuous variables that were normally distributed and by percentages for categorical variables. Right-skewed data are presented as median and 25 th to 75 th percentile. The baseline characteristics between patients alive and dead at 1-year were compared using chi-square tests for categorical variables and 1-way ANOVA for continuous variables. Event-free survival rates were determined using the Kaplan-Meier method, and survival curves were compared using the Log rank test. Kaplan-Meier curves was stratified by tertiles of each risk score for 1-year mortality. Receiver operating characteristic (ROC) curves were generated to measure the discrimination of the two models. The c-statistics was calculated and compared with the Wald test. To assess the model calibration, we created calibration plots for the SHFM and the MAGGIC score. The correlation between observed and predicted mortality was assessed using the Hosmer-Lemeshow (H-L) goodness-of-fit test (P-value $>0.05$ indicated the observed results are not statistically different from the predicted values). To assess the global performance of the two scores, absolute net reclassification improvement (NRI) was used.

A two-sided $\mathrm{P}<0.05$ was considered statistically significant. ROC curves were produced by MedCalc software v18.2.1 (MedCalc Software, Mariakerke, Belgium). Other analyses were performed using SPSS v23.0 (IBM, Armonk, NY, USA) and GraphPad Prism 8 (GraphPad, San Diego, California, USA).

\section{Results \\ Patient Characteristics}

Baseline characteristics are shown in Table 1. A total of 675 HF patients aged 70 years or older were enrolled in this study. The mean age was 80 years with $49.7 \%$ patients being $\geq 80$ years. Male patients accounted for $50.8 \%$. Of these elderly patients, $61.5 \%$ had preserved LVEF over $40 \%, 68.3 \%$ had an ischemic heart disease etiology, $92.2 \%$ were discharged on diuretics, $64.1 \%$ were being treated by either angiotensin-converting enzyme inhibitors (ACEI) or angiotensin receptor blockers (ARB), 76.9\% beta-blockers, and $62.2 \%$ spironolactone.

At 1-year follow-up, 74 patients (11.0\%) had died. As Table 1 shows, most covariates used in the risk scores were significantly different between those who were alive vs dead at 1-year follow-up. In particular, patients who died before 1-year follow-up were significantly older, had a lower weight, worse clinical status, and higher proportion of preserved LVEF. The use of ACEI and ARB was significantly lower in patients who died at 1-year follow-up and allopurinol use was higher. In this study, no patients used sacubitril/valsartan.

Patient characteristics by age group are presented in Table S1. With increasing age, patients were more likely to be female and to suffer atrial fibrillation. LVEF was preserved in $64.1 \%$ of the patients $\geq 80$ years old, compared with $27.3 \%$ in patients $<70$ years. HF-recommended medications were less used and diuretics were more widely used in patients $\geq 80$ compared with patients $<70$ years.

\section{Prognostic Analysis Based on Risk Scores} During the mean follow-up time of 32.6 \pm 20.2 months, 193 of 675 patients died (28.6\%). The cumulative 1-year mortality was $10.5 \%$ and 3 -year mortality was $26.1 \%$ for the elderly HF patients in this study. The predicted median 1-year mortality was $8.0 \%$ by the SHFM and $17.5 \%$ by the MAGGIC score. There was significant overprediction of mortality by the MAGGIC score and some underpredicted mortality by the SHFM for the entire cohort.

A Kaplan-Meier survival curve was drawn for the three risk categories divided by the risk scores and compared by a Log rank test (Figure 2). The event rates of allcause mortality significantly increased with increasing 
Table I Baseline Characteristics of Elderly Patients with Heart Failure by Status at I-Year Follow-Up

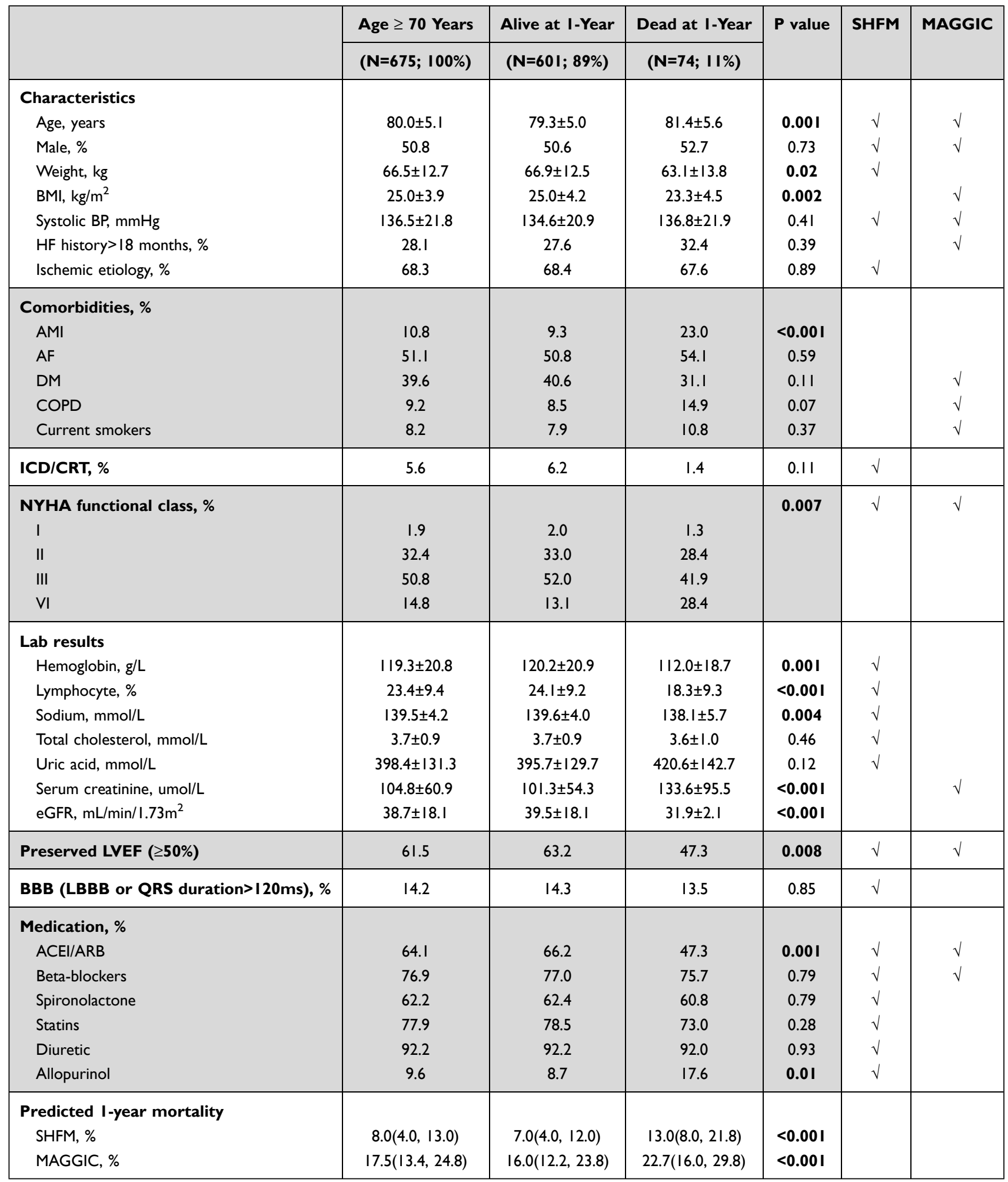

Notes: Values are mean \pm standard deviation, \%, or median (interquartile range); Statistical significance $(P<0.05)$ is indicated in bold text; $\sqrt{ }$ Components in the SHFM or MAGGIC risk scores.

Abbreviations: ACE, angiotensin-converting enzyme; AF, atrial fibrillation; AMI, acute myocardial infarction; ARB, angiotensin II receptor blocker; BMI, body mass index; BP, blood pressure; COPD, chronic obstructive pulmonary disease; CRT, cardiac resynchronization therapy; DM, diabetes mellitus; eGFR, estimated glomerular filtration rate; HF, heart failure; ICD, implantable cardioverter-defibrillator; LBBB, left bundle branch block; LVEF, left ventricular ejection fraction; MAGGIC, Meta-analysis Global Group in Chronic Heart Failure; NYHA, New York Heart Association functional class; SHFM, Seattle Heart Failure Model; TC, total cholesterol. 


\section{A}

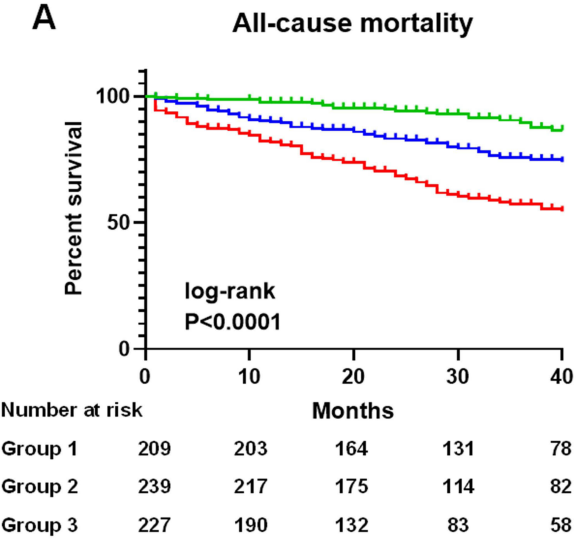

B
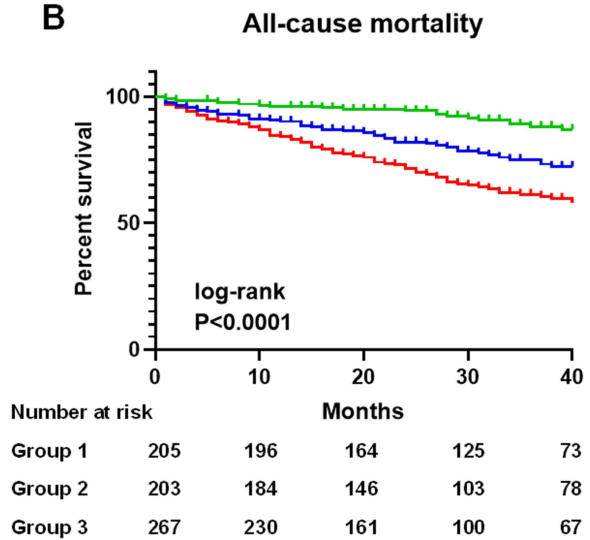

Figure 2 Kaplan-Meier curves of all-cause mortality stratified by tertiles of the SHFM (A) and MAGGIC (B) scores. The two risk scores were significantly associated with mortality (log-rank $\mathrm{P}<0.000 \mathrm{I}$ for all survival curves).

Abbreviations: MAGGIC, Meta-analysis Global Group in Chronic Heart Failure; SHFM, Seattle Heart Failure Model.

SHFM (Figure 2A) and MAGGIC (Figure 2B) score severity in all subjects (log-rank, $\mathrm{P}<0.0001$, respectively).

\section{Discrimination in Elderly Patients and Different Age Groups}

ROC analyses using c-statistics for each model showed that the SHFM provided better discrimination for 1-year mortality than the MAGGIC score in HF patients $\geq 70$ years old (SHFM, c-statistics $=0.73$; MAGGIC, c-statistics $=0.67 ; \mathrm{P}=0.05$ ) (Figure 3).

We compared the discriminatory capacity of each score in all ages and different age groups (Table 2). The SHFM showed significantly better discrimination than the MAGGIC score for HF patients of all ages (SHFM, c-statistic $=0.75 ;$ MAGGIC, $\mathrm{c}$-statistic $=0.69 ; \mathrm{P}=0.009)$. In patients $\geq 80$ years old, both risk scores were inaccurate in predicting 1-year mortality (SHFM, c-statistics $=0.65$; MAGGIC, c-statistics $=0.60 ; \mathrm{P}=0.09$ ).

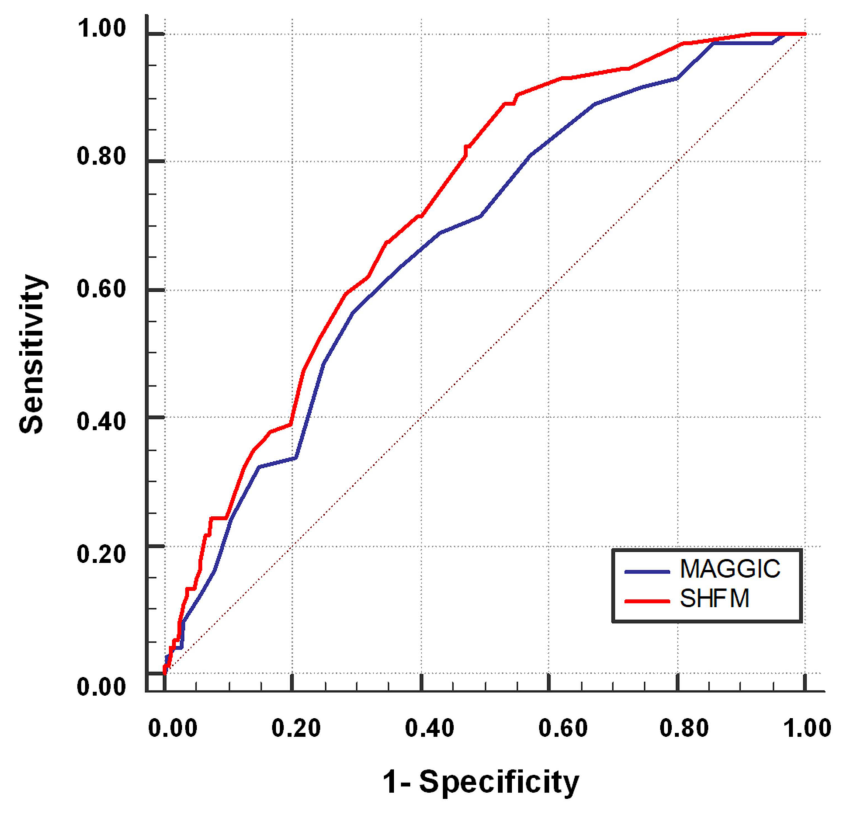

Figure 3 Receiver operating characteristic curves comparing the areas under the curve of risk scores for I-year mortality.

Abbreviations: MAGGIC, Meta-analysis Global Group in Chronic Heart Failure; SHFM, Seattle Heart Failure Model.

The discriminatory capacity of each score was compared in male vs female patients in this elderly HF population. The SHFM showed significantly better discrimination than the MAGGIC score in female patients (SHFM, c-statistics $=0.77$; MAGGIC, c-statistics $=0.65 ; \mathrm{P}=0.006$ ).

\section{Calibration and Risk Reclassification Analysis}

Next, we analyzed the calibration plots for 1-year mortality shown in Figure 4 . We found that the SHFM was generally well calibrated. Both risk scores showed overestimation for lower-risk groups and underestimation for higher-risk groups, a difference which appeared more extreme for the MAGGIC score. The Hosmer-Lemeshow goodness-of-fit (H-L) test was $>0.05$ for the SHFM, which confirms its ability to predict 1 -year mortality in elderly HF patients (H-L test, $\mathrm{P}=0.41$ for SHFM, $\mathrm{P}=0.02$ for MAGGIC). Based on the NRI analysis, the SHFM score showed better risk classification compared with the MAGGIC score (absolute NRI=2.96\%, P $<0.0001$; Figure 5).

\section{Discussion}

This is the first study to externally validate and compare the SHFM and MAGGIC scores in a real-world population of elderly Chinese HF patients. In this retrospective study, 
Table 2 Comparison of C-Statistic of the SHFM versus the MAGGIC Score for I-Year Mortality in Different Age Groups

\begin{tabular}{|c|c|c|c|}
\hline & \multicolumn{2}{|c|}{ C-Statistic $(95 \% \mathrm{Cl})$} & \multirow[t]{2}{*}{ P value } \\
\hline & SHFM & MAGGIC & \\
\hline All ages & $0.75(0.72-0.78)$ & $0.69(0.66-0.72)$ & 0.009 \\
\hline \multicolumn{4}{|l|}{ Three age categories } \\
\hline$\geq 80$ years & $0.65(0.6 I-0.7 I)$ & $0.60(0.55-0.66)$ & 0.09 \\
\hline $70-80$ years & $0.76(0.72-0.8 \mathrm{I})$ & $0.70(0.65-0.75)$ & 0.2 \\
\hline$<70$ years & $0.79(0.74-0.83)$ & $0.76(0.7 \mathrm{I}-0.8 \mathrm{I})$ & 0.62 \\
\hline
\end{tabular}

Note: Statistical significance $(P<0.05)$ is indicated in bold text.

Abbreviations: ACE, angiotensin-converting enzyme; AF, atrial fibrillation; AMI, acute myocardial infarction; ARB, angiotensin II receptor blocker; BMI, body mass index; BP, blood pressure; COPD, chronic obstructive pulmonary disease; CRT, cardiac resynchronization therapy; DM, diabetes mellitus; eGFR, estimated glomerular filtration rate; HF, heart failure; ICD, implantable cardioverter-defibrillator; LBBB, left bundle branch block; LVEF, left ventricular ejection fraction; MAGGIC, Meta-analysis Global Group in Chronic Heart Failure; NYHA, New York Heart Association functional class; SHFM, Seattle Heart Failure Model; TC, total cholesterol.

we showed that the performance of the SHFM score was superior in elderly Chinese HF patients owing to better discrimination, calibration and risk classification. The MAGGIC score had modest capacity for discrimination, but it showed poor calibration. In patients $\geq 80$ years old, both risk scores performed poorly in prediction of 1-year mortality.

Differences in HF patient characteristics and prognosis among geographical regions are of concern. ${ }^{15,16}$ As a middle-income country, China has a large number of HF patients, but epidemiological data is limited, especially for the elderly. In this real-world retrospective study, the mean age of the study population was 80 years with $49.7 \%$

\section{A SHFM Calibration Plots}

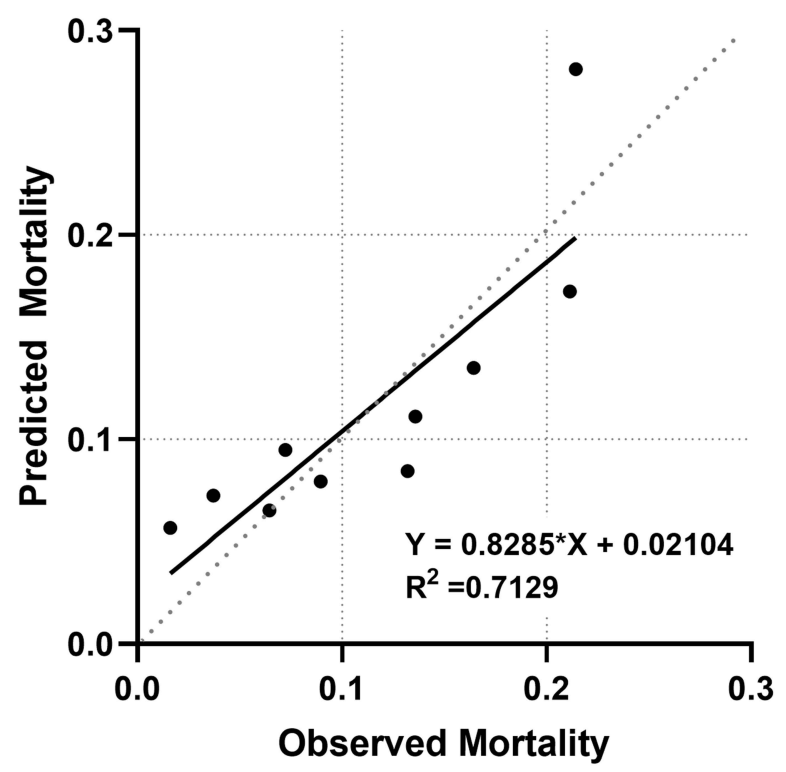

of patients being $\geq 80$ years old. We demonstrated that elderly Chinese patients with HF were more often female and had a higher prevalence of HFpEF and comorbidities, consistent with findings in studies of Western countries. $^{17,18}$

In this study, the use of beta-blockers (76.9\%) was similar to that in the ASIAN-HF Registry ${ }^{19}$ but lower than in Western countries. ${ }^{15,20}$ ACE inhibitors or ARBs were prescribed to $64.1 \%$ and spironolactone to $62.2 \%$ of the elderly patients, which was significantly lower than in the above studies. Our study indicated that prescription of evidence-based medications decreased with increasing age, whereas that of diuretics increased, consistent with

\section{B MAGGIC Calibration Plots}

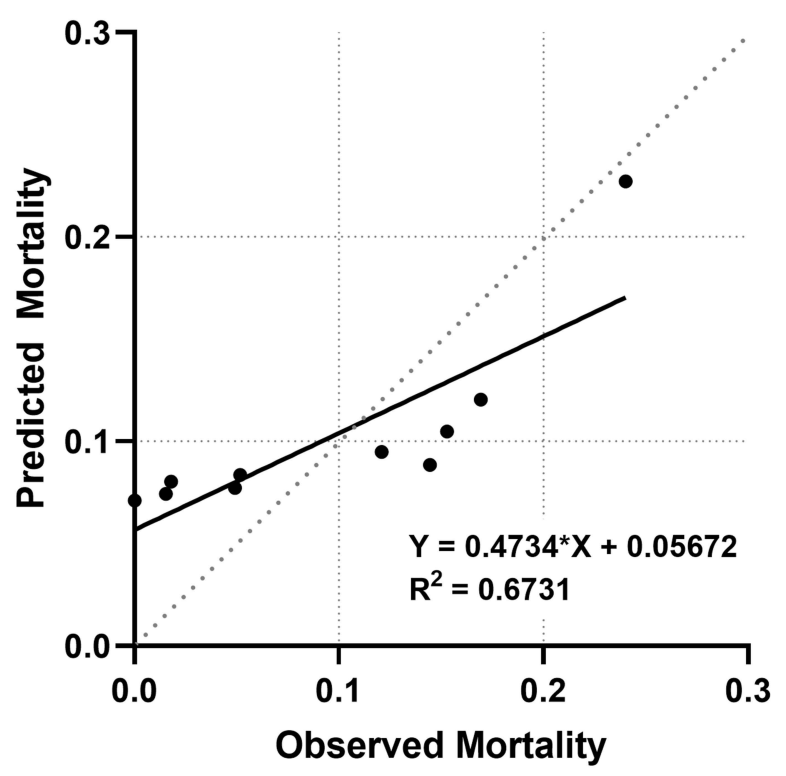

Figure 4 I-year observed probabilities of mortality at different risk deciles in the overall population predicted by (A) the Seattle Heart Failure Model (SHFM) and (B) the Meta-Analysis Global Group in Chronic Heart Failure (MAGGIC) score. The diagonal dashed lines represent perfect calibration. 


\begin{tabular}{|c|c|c|c|}
\hline \multicolumn{4}{|c|}{ Patients dead at 1-year $(\mathrm{n}=74)$} \\
\hline & \multicolumn{3}{|c|}{ SHFM } \\
\hline MAGGIC & Lower risk & Higher risk & Total \\
\hline Lower risk & 9 & 12 & 21 \\
\hline Higher risk & 5 & 48 & 53 \\
\hline Total & 14 & 60 & 74 \\
\hline \multicolumn{4}{|c|}{ SHFM } \\
\hline \multicolumn{4}{|c|}{ Patients alive at 1-year (601) } \\
\hline MAGGIC & Lower risk & Higher risk & Total \\
\hline Lower risk & 230 & 74 & 317 \\
\hline Higher risk & 87 & 210 & 284 \\
\hline Total & 317 & 284 & 601 \\
\hline \multicolumn{4}{|c|}{ Absolute NRI $=2.96 \%, \mathrm{Z}=5.88, \mathrm{P}<0.0001$} \\
\hline
\end{tabular}

Figure 5 Risk reclassification of the MAGGIC versus SHFM score for I-year mortality in elderly heart failure patients.

Abbreviations: MAGGIC, Meta-analysis Global Group in Chronic Heart Failure; SHFM, Seattle Heart Failure Model; NRI, net reclassification improvement.

the EHFS II survey ${ }^{21}$ and CHART-2 study. ${ }^{18}$ This underscores the underuse of cardioprotective medications in elderly Chinese. On the other hand, this underuse may be the result of therapies recommended in current guidelines having not been adequately tested in elderly patients. The benefits of these drugs in elderly HF patients, particularly in those with HFpEF, are uncertain.

Owing to the distinctive characteristics of elderly HF patients, the predictors of mortality in elderly patients are different from those in young patients. Decreased EF is a strong predictor of death for the young. However, for elderly patients, decreased EF was not an independent predictor. $^{21}$ For elderly patients, comorbidities, such as anemia and diabetes mellitus (DM), were more associated with long-term mortality. ${ }^{7}$ Owing to the distinctive characteristics and poorer prognosis of elderly patients, developing targeted risk assessment for them is important. In this study, we externally validated and compared the SHFM and MAGGIC scores in a cohort of elderly Chinese HF patients.

The SHFM was derived from a cohort of only about 1000 HFrEF patients with a mean age of 65 years. ${ }^{10}$ The SHFM is the most widely used prognostic model for HF and has been validated in a wide variety of patients. ${ }^{22-24}$ The discrimination capacity of the SHFM in external validation cohorts has varied from poor to acceptable (c-statistics, $0.63-$ 0.81 ), and the model calibration showed a high correlation between observed and predicted survival. ${ }^{9}$ In the present study, the SHFM provided for good discrimination for 1 -year mortality in elderly HF patients (c-statistics $=0.73$ ) and adequate model goodness-of-fit as assessed by calibration plots and the Hosmer-Lemeshow test. The SHFM underestimated mortality risk in our elderly HF cohort ( $8.0 \%$ for predicted 1 -year mortality vs $10.5 \%$ for actual 1-year mortality), consistent with the results of $\mathrm{Li}$ et al. ${ }^{14}$

The MAGGIC meta-analysis ${ }^{11}$ included 39,372 ambulatory HF patients from 30 studies with reduced or preserved LVEF. The mean age of the MAGGIC cohort was 68 years, older than in the SHFM cohort. The MAGGIC score is increasingly used and well validated in $\mathrm{HFpEF}$ and Asian HF patients. ${ }^{25-27}$ The MAGGIC score in external validation cohorts showed acceptable discrimination capacity (c-statistics, 0.69-0.74) and model calibration. ${ }^{23,27}$ In the elderly Chinese HF population considered here, the MAGGIC score provided only modest discrimination for 1-year mortality (c-statistics $=0.67$ ) and inadequate model calibration with overprediction of mortality for the low-risk group and 
underprediction for the high-risk group. Our results suggest that the SHFM score is perhaps more appropriate for guiding clinical interventions for elderly Chinese HF patients because it exhibited better discrimination, calibration and risk classification than did the MAGGIC score. This may result from the SHFM score having more medical data that represents non-cardiac comorbidities, including hemoglobin and sodium levels, which are predictors of mortality in the elderly. ${ }^{28,29}$ However, both scores performed poorly in predicting 1 -year mortality for patients $\geq 80$ years old. Frailty is a biological syndrome characterized by decreased reserve and resistance to stressors, which is prevalent in elderly HF patients and can affect quality of life in the elderly. ${ }^{30}$ In the future, we may build a new risk score that also includes a variable for frailty.

Women with HF differ from men in several respects. Women are more likely to be HFpEF, less ischemic and a higher proportion have hypertension and DM. ${ }^{31}$ In agreement with a recent study, ${ }^{32}$ the SHFM in the present study showed significantly better discriminatory capacity in women, which may be because the SHFM contains variables related to multiple underlying diseases.

Our work has several limitations that should be acknowledged. First, this was a small single-center retrospective study and therefore has several inherent limitations, including selection bias. This population may not be representative of the entire population of elderly Chinese HF patients. Second, the two scores selected here were designed for ambulatory HF patients, but our study included patients discharged from hospitals who have a higher proportion of comorbidities and a poorer prognosis. Third, because our study lacked variables from cardiopulmonary exercise tests, we could not test additional prognostic scores.

\section{Conclusion}

In conclusion, the SHFM has good discrimination, calibration and risk classification for prediction of 1-year mortality in elderly Chinese HF patients, and it performs better than the MAGGIC score. For patients $\geq 80$ years old, both risk scores perform poorly in predicting mortality.

\section{Abbreviations}

$\mathrm{ACE}$, angiotensin-converting enzyme; AF, atrial fibrillation; AMI, acute myocardial infarction; ARB, angiotensin II receptor blocker; BMI, body mass index; BP, blood pressure; COPD, chronic obstructive pulmonary disease; CRT, cardiac resynchronization therapy; DM, diabetes mellitus; eGFR, estimated glomerular filtration rate; HF, heart failure; ICD, implantable cardioverter-defibrillator; LBBB, left bundle branch block; LVEF, left ventricular ejection fraction; MAGGIC, Meta-analysis Global Group in Chronic Heart Failure; NYHA, New York Heart Association functional class; SHFM, Seattle Heart Failure Model; TC, total cholesterol.

\section{Funding}

The study has not been funded.

\section{Disclosure}

The authors declare that they have no known competing financial interests or personal relationships that could have appeared to influence the work reported in this paper.

\section{References}

1. Chen J, Normand S-LT, Wang Y, Krumholz HM. National and regional trends in heart failure hospitalization and mortality rates for Medicare beneficiaries, 1998-2008. JAMA. 2011;306 (15):1669-1678. doi:10.1001/jama.2011.1474

2. Bleumink GS, Knetsch AM, Sturkenboom MCJM, et al. Quantifying the heart failure epidemic: prevalence, incidence rate, lifetime risk and prognosis of heart failure The Rotterdam Study. Eur Heart J. 2004;25(18):1614-1619. doi:10.1016/j.ehj.2004.06.038

3. Pocock SJ, Wang D, Pfeffer MA, et al. Predictors of mortality and morbidity in patients with chronic heart failure. Eur Heart J. 2006;27 (1):65-75. doi:10.1093/eurheartj/ehi555

4. Siirilä-Waris K, Lassus J, Melin J, Peuhkurinen K, Nieminen MS, Harjola V-P. Characteristics, outcomes, and predictors of 1-year mortality in patients hospitalized for acute heart failure. Eur Heart $J$. 2006;27(24):3011-3017. doi:10.1093/eurheartj/ehl407

5. Ponikowski P, Voors AA, Anker SD, et al. 2016 ESC Guidelines for the diagnosis and treatment of acute and chronic heart failure: the Task Force for the diagnosis and treatment of acute and chronic heart failure of the European Society of Cardiology (ESC)Developed with the special contribution of the Heart Failure Association (HFA) of the ESC. Eur Heart J. 2016;37(27):2129-2200. doi:10.1093/eurheartj/ ehw128

6. Mogensen UM, Ersbøll M, Andersen M, et al. Clinical characteristics and major comorbidities in heart failure patients more than 85 years of age compared with younger age groups. Eur J Heart Fail. 2011;13 (11):1216-1223. doi:10.1093/eurjhf/hfr116

7. Barsheshet A, Shotan A, Cohen E, et al. Predictors of long-term (4-year) mortality in elderly and young patients with acute heart failure. Eur J Heart Fail. 2010;12(8):833-840. doi:10.1093/eurjhf/ hfq079

8. Howlett JG. Should we perform a heart failure risk score? Circ Heart Fail. 2013;6(1):4-5. doi:10.1161/CIRCHEARTFAILURE.112.973172

9. Alba AC, Agoritsas T, Jankowski M, et al. Risk prediction models for mortality in ambulatory patients with heart failure: a systematic review. Circ Heart Fail. 2013;6(5):881-889. doi:10.1161/ CIRCHEARTFAILURE.112.000043

10. Levy WC, Mozaffarian D, Linker DT, et al. The Seattle Heart Failure Model: prediction of survival in heart failure. Circulation. 2006;113 (11):1424-1433. doi:10.1161/CIRCULATIONAHA.105.584102

11. Pocock SJ, Ariti CA, McMurray JJV, et al. Predicting survival in heart failure: a risk score based on 39372 patients from 30 studies. Eur Heart J. 2013;34(19):1404-1413. doi:10.1093/eurheartj/ehs337 
12. Chinese Society of Cardiology of Chinese Medical Association. Chinese guidelines for the diagnosis and treatment of heart failure 2014. Zhonghua Xin Xue Guan Bing Za Zhi. 2014;42(2):98-122.

13. Sawano M, Shiraishi Y, Kohsaka S, et al. Performance of the MAGGIC heart failure risk score and its modification with the addition of discharge natriuretic peptides. ESC Heart Fail. 2018;5 (4):610-619. doi:10.1002/ehf2.12278

14. Li S, Marcus P, Núñez J, Núñez E, Sanchis J, Levy WC. Validity of the Seattle Heart Failure Model after heart failure hospitalization. ESC Heart Fail. 2019;6(3):509-515. doi:10.1002/ehf2.12427

15. Crespo-Leiro MG, Anker SD, Maggioni AP, et al. European Society of Cardiology Heart Failure Long-Term Registry (ESC-HF-LT): 1-year follow-up outcomes and differences across regions. Eur $J$ Heart Fail. 2016;18(6):613-625. doi:10.1002/ejhf.566

16. Groenewegen A, Rutten FH, Mosterd A, Hoes AW. Epidemiology of heart failure. Eur J Heart Fail. 2020;22(8):1342-1356. doi:10.1002/ ejhf. 1858

17. Manzano L, Babalis D, Roughton M, et al. Predictors of clinical outcomes in elderly patients with heart failure. Eur J Heart Fail. 2011;13(5):528-536. doi:10.1093/eurjhf/hfr030

18. Sato M, Sakata Y, Sato K, et al. Clinical characteristics and prognostic factors in elderly patients with chronic heart failure -A report from the CHART-2 study. Int J Cardiol Heart Vasc. 2020;27:100497. doi:10.1016/j.ijcha.2020.100497

19. Teng T-HK, Tromp J, Tay WT, et al. Prescribing patterns of evidence-based heart failure pharmacotherapy and outcomes in the ASIAN-HF registry: a cohort study. The Lancet Global Health. 2018;6(9):e1008-e1018. doi:10.1016/S2214-109X(18)30306-1

20. Fonarow GC, Albert NM, Curtis AB, et al. Improving evidence-based care for heart failure in outpatient cardiology practices: primary results of the Registry to Improve the Use of Evidence-Based Heart Failure Therapies in the Outpatient Setting (IMPROVE HF). Circulation. 2010;122(6):585-596. doi:10.1161 CIRCULATIONAHA.109.934471

21. Komajda M, Hanon O, Hochadel M, et al. Contemporary management of octogenarians hospitalized for heart failure in Europe: euro Heart Failure Survey II. Eur Heart J. 2009;30(4):478-486. doi:10.1093/eurheartj/ehn539

22. Canepa M, Fonseca C, Chioncel O, et al. Performance of Prognostic Risk Scores in Chronic Heart Failure Patients Enrolled in the European Society of Cardiology Heart Failure Long-Term Registry. JACC Heart Fail. 2018;6(6):452-462. doi:10.1016/j.jchf.2018.02.001

23. Allen LA, Matlock DD, Shetterly SM, et al. Use of Risk Models to Predict Death in the Next Year Among Individual Ambulatory Patients With Heart Failure. JAMA Cardiol. 2017;2(4):435-441. doi:10.1001/jamacardio.2016.5036
24. Freitas P, Aguiar C, Ferreira A, Tralhão A, Ventosa A, Mendes M. Comparative Analysis of Four Scores to Stratify Patients With Heart Failure and Reduced Ejection Fraction. Am J Cardiol. 2017;120 (3):443-449. doi:10.1016/j.amjcard.2017.04.047

25. Sartipy U, Dahlström U, Edner M, Lund LH. Predicting survival in heart failure: validation of the MAGGIC heart failure risk score in 51,043 patients from the Swedish heart failure registry. Eur J Heart Fail. 2014;16(2):173-179. doi:10.1111/ejhf.32

26. Rich JD, Burns J, Freed BH, Maurer MS, Burkhoff D, Shah SJ. Meta-Analysis Global Group in Chronic (MAGGIC) Heart Failure Risk Score: validation of a Simple Tool for the Prediction of Morbidity and Mortality in Heart Failure With Preserved Ejection Fraction. J Am Heart Assoc. 2018;7(20):e009594. doi:10.1161/ JAHA.118.009594

27. Khanam SS, Choi E, Son J-W, et al. Validation of the MAGGIC (Meta-Analysis Global Group in Chronic Heart Failure) heart failure risk score and the effect of adding natriuretic peptide for predicting mortality after discharge in hospitalized patients with heart failure. PLoS One. 2018;13(11):e0206380. doi:10.1371/journal. pone. 0206380

28. Park JJ, Cho Y-J, Oh I-Y, et al. Short and long-term prognostic value of hyponatremia in heart failure with preserved ejection fraction versus reduced ejection fraction: an analysis of the Korean Acute Heart Failure registry. Int J Cardiol. 2017;248:239-245. doi:10.1016/ j.ijcard.2017.08.004

29. Caughey MC, Avery CL, Ni H, et al. Outcomes of patients with anemia and acute decompensated heart failure with preserved versus reduced ejection fraction (from the ARIC study community surveillance). Am J Cardiol. 2014;114(12):1850-1854. doi:10.1016/ j.amjcard.2014.09.024

30. Dewan P, Jackson A, Jhund PS, et al. The prevalence and importance of frailty in heart failure with reduced ejection fraction - an analysis of PARADIGM-HF and ATMOSPHERE. Eur J Heart Fail. 2020;22 (11):2123-2133. doi:10.1002/ejhf.1832

31. Kajimoto K, Minami Y, Otsubo S, Sato N. Sex Differences in Left Ventricular Cavity Dilation and Outcomes in Acute Heart Failure Patients With Left Ventricular Systolic Dysfunction. Can J Cardiol. 2018;34(4):477-484. doi:10.1016/j.cjca.2018.01.019

32. Vishram-Nielsen JKK, Foroutan F, Ross HJ, Gustafsson F, Alba AC. Performance of Prognostic Risk Scores in Heart Failure Patients: do Sex Differences Exist? Can J Cardiol. 2020;36(1):45-53. doi:10.1016/j.cjca.2019.08.021
Clinical Interventions in Aging

\section{Publish your work in this journal}

Clinical Interventions in Aging is an international, peer-reviewed journal focusing on evidence-based reports on the value or lack thereof of treatments intended to prevent or delay the onset of maladaptive correlates of aging in human beings. This journal is indexed on PubMed Central, MedLine, CAS, Scopus and the Elsevier

\section{Dovepress}

Bibliographic databases. The manuscript management system is completely online and includes a very quick and fair peer-review system, which is all easy to use. Visit http://www.dovepress.com/ testimonials.php to read real quotes from published authors. 